Ann. Biol. anim. Bioch. Biophys., I969, 9 (3), 369-37٪.

\title{
CONSEQUENCES DE L'INTRODUCTION DU GÈNE DE NANISME $d w$ SUR L'UTILISATION ALIMENTAIRE CHEZ LE POUSSIN FEMELLE
}

\author{
J. GUILLAUME \\ avec la collaboration technique de Danielle Bouil.lon et Liliane Croizier-Robin \\ Station de Recherches avicoles, \\ Centre de Recherches de Tours, 37 - Nouzilly \\ Institut national de la Recherche agronomique
}

SOMMAIRE

On compare l'utilisation d'un même régime chez des poulettes sœurs normales ou naines (allèle $d w$ ) entre o et 9 semaines. Outre une croissance ralentie, les sujets nains se caractérisent par une mauvaise rétention azotée et un mauvais indice de consommation malgré une réduction de leur besoin d'entretien et de leur métabolisme au repos. Les tissus synthétisés par les naines contiennent davantage de lipides et le rapport énergie stockée/énergie métabolisable ingérée est en leur faveur. L'énergie métabolisable n'est pas affectée par le gène $d w$. Le comportement relativement boulimique et les caractères hypothyroïliens des naines sont discutés.

\section{INTRODUCTION}

Le gène $d w$ découvert par HuTT (I953) est un gène de nanisme récessif lié au sexe qui réduit le poids de la poule de $30 \mathrm{p}$. Ioo et celui du coq de $40 \mathrm{p}$. Ioo environ. Il provoque une consommation moindre d'aliment chez la pondeuse mais il élève relativement ses besoins en calcium (BERNIER et ARscotT, I96o ; ProD'HOMmE et MÉRAT, I969) et en protéines (ARSCOTT et BERNIER, I968) lorsqu'on les exprime en pourcentage du régime. Chez le jeune, à notre connaissance, aucune étude de nutrition comparée n'a été publiée à l'exception de celle concernant l'indice de consommation de poulettes Leghorn naines ou normales (BERNIER et ARSCOTT, I966).

Nous rapportons les résultats d'une expérience effectuée sur des poulettes en croissance portant l'allèle $\mathrm{D} w$ ou $d w$ en vue d'étudier l'influence éventuelle du gène sur l'utilisation de l'énergie, des protéines et de la ration globale. 


\section{MATÉRIEL, ET TECHNIQUES}

Les ${ }^{3} 8$ animaux mis en expérience sont des poussins issus d'un croisement pédigré coq hétérozygote $X$ femelle normale, autosexable par ailleurs grâce au gène $K$. T'ous les mâles sont éliminés tandis que les femelles dont le génotype est $d w-$ ou $\mathrm{D} w$ - dans la proportion de $5 \circ \mathrm{p}$. I00-5० p. I00 sont conservées et sont élevées en cages individuelles. Le génotype est déterminé en fin d'expérience (9 semaines) et à ce moment des comparaisons sont faites, soit sur les couples de vraies sœurs, soit sur les couples de vraies et demi-sœurs.

On enregistre l'évolution du poids et de la consommation hebdomadaire de chaque animal. En outre, sur un groupe de I 2 poulettes choisies au hasard à raison de 2 par famille, on effectue des bilans et des mesures d'énergie métabolisable sur des périodes de 3 jours à deux stades de croissance. A la fin de l'expérience (9 semaines) I4 paires de vraies sœurs et 9 de demi-sœurs sont sacrifiées en vue de l'analyse corporelle. I4 poulettes choisies au hasard à raison de 2 par famille servent à des mesures de consommation d'oxygène à divers stades de croissance entre I et I I semaines.

Pour effectuer l'analyse corporelle, nous dosons les matières grasses par la méthode de DELPECH et al. (1966) et l'azote à l'auto-analyseur TECHNICON. L'énergie métabolisable est déterminée selon la technique de HILL et ANDERSON (I958). La consommation d'oxygène est mesurée en deçà de 3 semaines sur des périodes de $\mathrm{x} 2$ heures environ à l'aide d'un appareil en circuit fermé (GuILlaume, r 969) et au-delà sur des périodes d'une heure à l'aide d'un appareil en circuit ouvert muni d'un analyseur automatique à susceptibilité paramagnétique et d'un enregistreur potentiométrique.

Les régimes utilisés sont des aliments usuels dont les caractéristiques principales sont rassemblées dans les tableaux I et 2. Le régime de "démarrage " est remplacé par le régime de "finition " à l'âge de 5 semaines.

\section{RÉSULTATS}

On enregistre une mortalité de $5 \mathrm{p}$. Ioo environ au cours de la première semaine où certains animaux s'adaptent mal à la vie en cages individuelles.

$\mathrm{Du} \mathrm{ro}^{\mathrm{e}}$ au $30^{\mathrm{e}}$ jour, un nombre considérable de boiteries apparaît. Dans la majeure partie des cas il s'agit de perosis plus ou moins grave. La cause de cette anomalie est recherchée ; l'aliment après analyse s'avère avoir des teneurs normales en calcium, phosphore, choline, et être très largement excédentaire en manganèse ( $I, I_{4}$ p. Ioo, $0,68 \mathrm{p}$. I00, $0,27 \mathrm{p}$. IOo et $340 \mathrm{mg}$ au $\mathrm{kg}$ respectivement). Une administration de toutes les vitamines hydrosolubles dans l'eau de boisson n'empêche pas l'augmentation de l'incidence de boiteries. L,es individus nains et normaux sont atteints peu à peu également ( $60 \mathrm{p}$. Ioo et $54 \mathrm{p}$. Ioo respectivement). Certaines familles cependant sont touchées à Ioo p. Ioo, tandis que 6 sur 27 restent totalement indemnes, ce qui suggère l'existence d'une composante génétique sans rapport avec le gène $d w$. Bien que la cause de cette apparition tout à fait anormale de perosis n'ait pu être déterminée, 23 paires de sœurs ou demi-sœurs totalement indemnes ou très légèrement atteintes sont utilisées pour l'analyse statistique des résultats qui, de ce fait, ne portent pas sur toutes les familles.

\section{Développement pondéral}

Les poussins, tous issus d'œufs de taille normale, ont un poids identique à la naissance qu'ils soient des allèles Dw ou $d w$. Au fur et à mesure que l'expérience se déroule les poussins $d w$ accusent un retard pondéral de plus en plus marqué sur les normaux (fig. I) : les taux de croissance $\left(\frac{\Delta P}{P}\right)$ des individus nains et normaux ne 


\section{TABLEAU I}

Composition de l'aliment de démxrrage 0 à 5 semaines

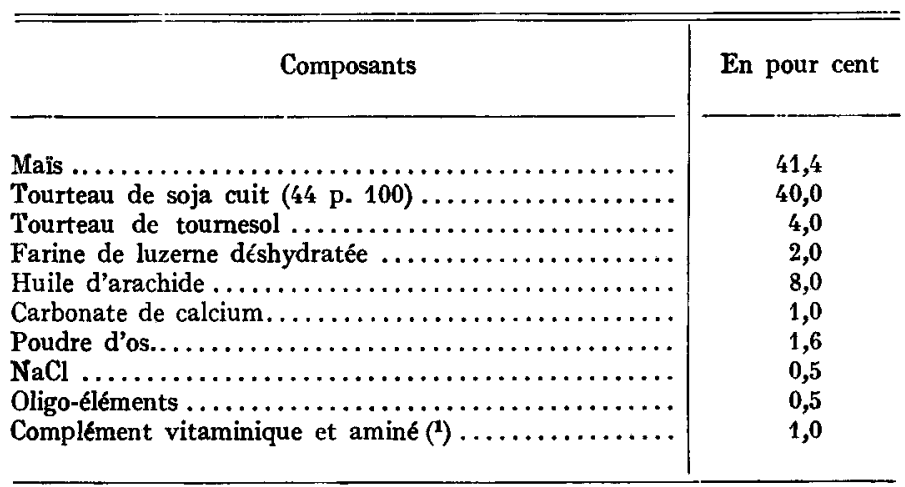

(1) Apportant pour $100 \mathrm{~kg}$ de régime : Vitamine A (UI) 800 000, Vitaminte $\mathrm{D}_{3}$ (UI) $100000, \alpha$-Tocophérol (à 25 p. 100) $20,0 \mathrm{~g}$, Riboflavine $0,3 \mathrm{~g}$, Pantothénate de calcium 0,5 g, Acide nicotinique $1,0 \mathrm{~g}$, Vitamine $B_{12}$ (à $1 /$ 10 000) 0,1 g, Choline (à 25 p. 100) 400,0 g, Méthionine D.L. 150,0 g, Sulfate de manganèse $35,0 \mathrm{~g}$, B.H.I'. 12,5 g, Amprolium (à 25 p. 100) 40,0 g, $\alpha$-Tocophérol (à 25 p. 100) 20,0 g, cérélose $320,0 \mathrm{~g}$.

TABLEAU 2

Composition de l'aliment de finition de 5 à 9 semaines

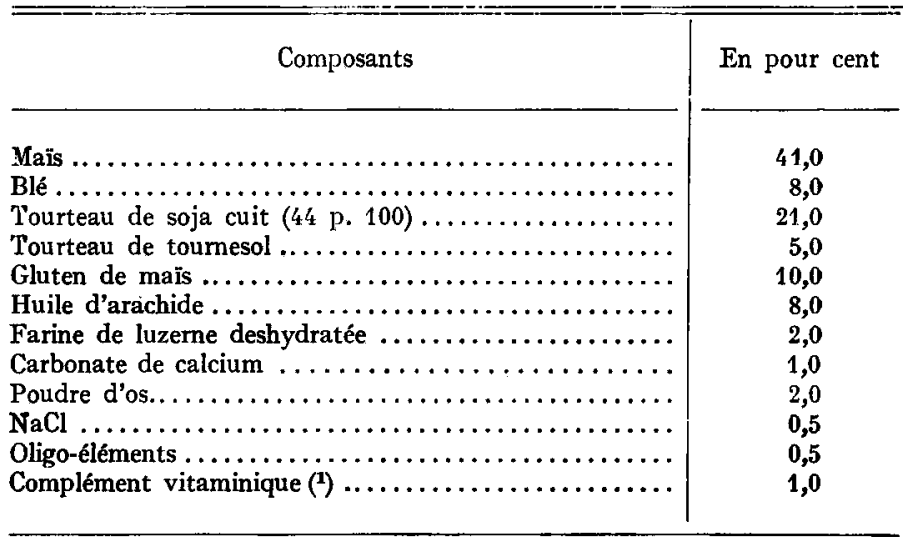

(1) Apportant pour $100 \mathrm{~kg}$ de régime : Vitamine A (UI) 800 000, Vitamine $D_{\mathrm{a}}$ (UI) $100000, \alpha$-Tocophérol $20,0 \mathrm{~g}$, Riboflavine $0,3 \mathrm{~g}$, Choline (à 25 p. 100) $400,0 \mathrm{~g}$, Sulfate de manganèse $35,0 \mathrm{~g}$, B.H.T. $12,5 \mathrm{~g}$, Amprolium. (à 25 p. 100) 28,0 g, Avoine broyée 500,0 g. 
restent pas dans un rapport constant au cours de la croissance ; l'infériorité des " naines " vis-à-vis des " normales " s'accentue avec l'âge, de o à 9 semaines.

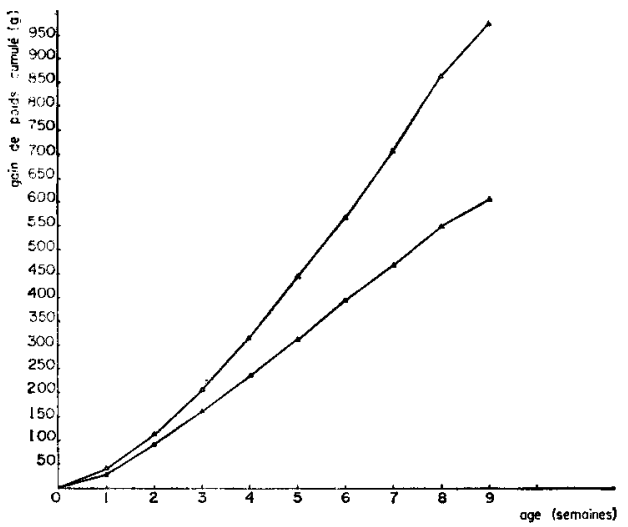

FIG. I. - Gain de poids cumulé de poulettes "naines " et "normales"

établi sur 8 paires de vraies saurs et 8 de demi-soeurs

$\longrightarrow \Delta-\Delta-$ " naines * $(d w) \quad \longrightarrow \Delta-\Delta-\Delta$ \& normales $(\mathrm{D} w)$

\section{Consommation}

La consommation des poulettes naines est toujours inférieure à celle des normales de même âge. Néanmoins, si on la rapporte au poids des poulettes, on s'aperçoit que les naines consomment à peu près autant que leurs sœurs normales (fig. 2). Pendant une période de 3 semaines seulement de la $5^{\mathrm{e}}$ à la $7^{\mathrm{e}}$ semaine incluse, le taux d'ingestion des " $d w$ » est momentanément inférieur à delui des « $\mathrm{D} w$ ».

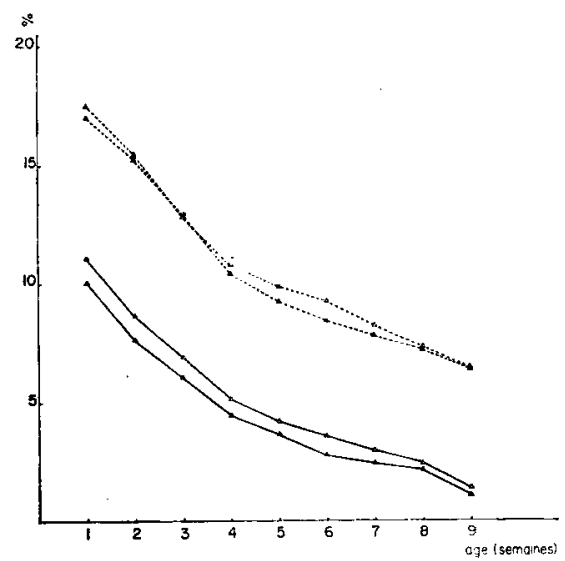

FIG. 2. - Taux de croissance et taux d'ingestion de poulettes " naines "el "normales " etabli sur 8 paires de vraies sours et 8 demi-sceurs taux d'ingestion - taux de croissance

Même convention que dans la figure i pour les symboles correspondant aux deux génotypes

\section{Rétention azotée et coefficient d'utilisation protéique}

Les bilans azotés, établis sur II animaux (I moribond ayant été éliminé), fournissent peu de renseignements intéressants car 4 poulettes seulement ont le géno- 
type $d w$. On peut vérifier que la rétention azotée est significativement plus faible chez les naines $(p<0,05)$ mais l'importance de la différence est difficile à chiffrer par suite de l'erreur d'échantillonnage.

Les analyses corporelles (tabl. 3) confirment ces résultats sur un plus grand nombre de couples et pour toute la durée de l'essai. La teneur azotée des carcasses en fin d'expérience n'est pas significativement modifiée par le gène $d w$; la rétention azotée et le coefficient d'utilisation azotée sont donc influencés de la même façon que le gain de poids et l'efficacité alimentaire respectivement.

\section{TABLEAU 3}

Composition corporelle et coefficient d'utilisation de l'azote et de l'énergie chez des poulettes "naines " et " normales"

(23 paires de sours)

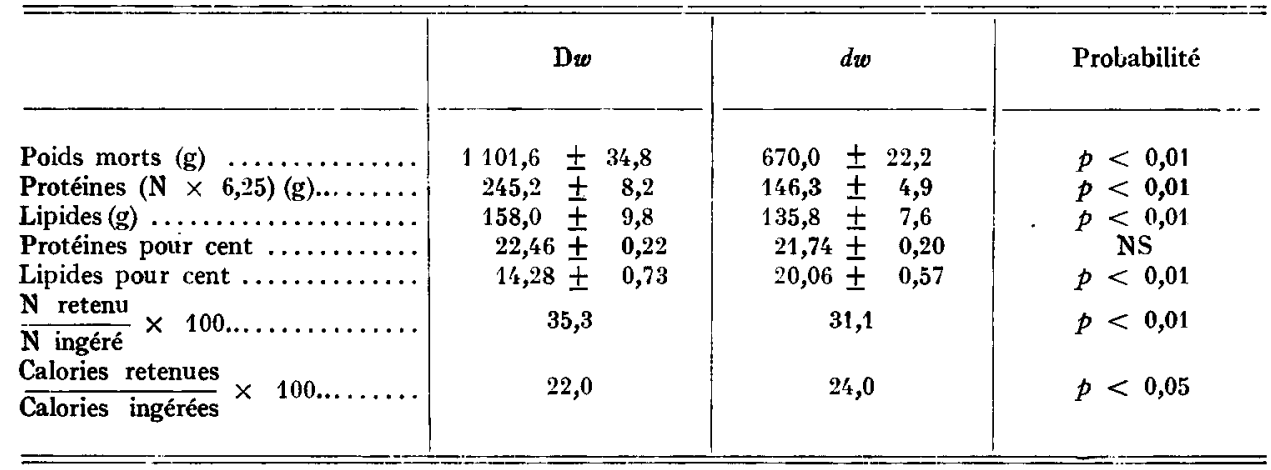

\section{Utilisation de l'énergie}

a) Énergie métabolisable.

Les mesures effectuées sur les II animaux précédents montrent que les valeurs d'énergie métabolisable, classique ou corrigée (tabl. 4) des aliments de démarrage ou

TABLEAU 4

Energie métabolisable des aliments

(kcal/g)

\begin{tabular}{|c|c|c|c|}
\hline Génotype $\ldots \ldots \ldots \ldots \ldots \ldots$ & $\mathbf{D} w(7)(\mathbf{l})$ & $d w(4)$ & Probabilité \\
\hline $\begin{array}{l}\text { Aliment " démarrage } " . . \ldots \ldots \ldots \\
\text { Aliment " finition } " . . . \ldots \ldots \ldots\end{array}$ & $\begin{array}{l}3,32 \pm 0,02 \\
3,53 \pm 0,02\end{array}$ & $\begin{array}{l}3,21 \pm 0,04 \\
3,49 \pm 0,03\end{array}$ & $\begin{array}{l}\text { NS } \\
\text { NS }\end{array}$ \\
\hline
\end{tabular}

(1) Entre parenthèses, nombre d'individus utilisés.

de finition sont les mêmes pour les deux groupes d'animaux. Aucune différence d'utilisation énergétique n'est donc imputable à des phénomènes d'ordre digestif. 
b) Métabolisme énergétique.

La consommation d'oxygène par unité de poids est représentée en fonction de l'âge sur la figure 3. Comparées aux poulettes normales, les naines ont un métabolisme plus intense, jusqu'à 9 semaines du moins. Cette différence demeure si l'on rapporte la consommation d'oxygène au poids vif élevé à la puissance 0,70 au lieu du poids vif. Toutefois, si l'on étudie le taux métabolique en fonction du poids et non de l'âge, une conclusion opposée se dégage : la consommation d'oxygène par unité de poids (ou de poids élevé à la puissance 0,70 ) est en tout point inférieure chez les naines. Ces résultats permettent de supposer que l'utilisation médiocre de l'aliment par les poussins dw n'est pas causée par une dépense énergétique exagérée : l'ensemble des dépenses du métabolisme basal et de l'extrachaleur est au contraire en leur faveur.
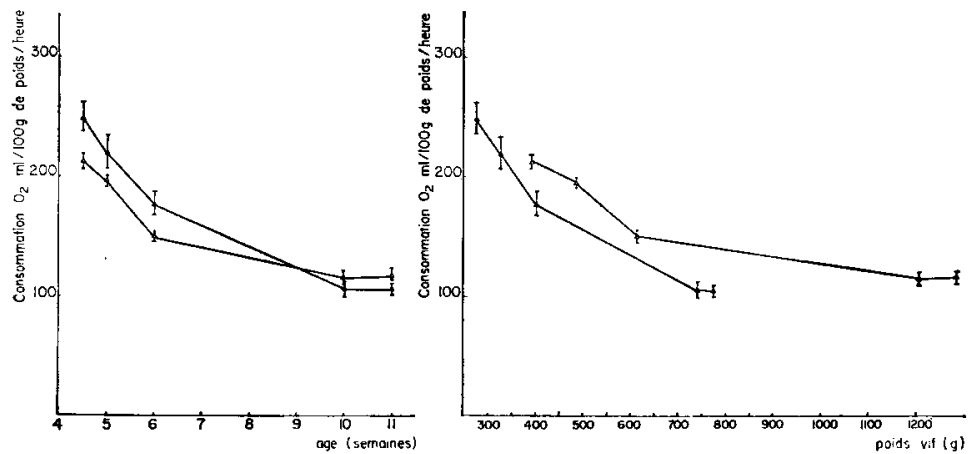

FIG. 3. - Consommation d'oxygène par unité de poids chez des poulettes "naines " et "normales " établi sur 7 couples de saurs

Même convention que sur la figure I pour les symboles correspondant aux deux génotypes

Les analyses corporelles (tabl. 3) révèlent des différences considérables de teneur en lipides et partant de valeur énergétique des carcasses : chaque gramme de poids gagné par un poussin représente $2 \mathrm{I}$ p. Ioo de plus de calories stockées chez un $d w$ que chez un D $w$. L'utilisation globale de l'énergie mesurée par le rapport :

énergie retenue énergie métabolisable ingérée s'avère en faveur des poulettes naines; elles utilisent donc l'aliment mieux que les normales si on mesure le gain par l'énergie stockée.

On peut noter enfin, même sur une faible période, que les enregistrements obtenus avec les deux groupes de poulettes ont des allures très différentes, les " dents de scie " étant beaucoup moins fréquentes et moins prononcées chez les poulettes naines. La dépense énergétique due aux mouvements y est donc plus faible et cela confirme l'impression subjective que les animaux sont d'un naturel extrêmement calme.

\section{Efficacité alimentaire globale. Entretien et croissance}

Consommant autant et croissant moins vite que les normales de même poids (mais d'âge différent), il est clair que les poulettes naines ont une moins bonne efficacité alimentaire. De ce fait, l'indice de consommation cumulé est nettement plus élevé $(p<0,0 I)$ chez ces dernières (fig. 4). La différence est de 0,1 à $0,2 \mathrm{~kg}$ d'aliment $/ \mathrm{kg}$ de poids vif gagné. 
Une exception intéressante est cependant à noter : lors de la Ire semaine, 1 'indice de consommation est similaire dans les deux groupes alors que le développement pondéral est déjà affecté par le gène $d w$. On peut attribuer cette exception au fait que durant leur première semaine de vie les poussins nains disposent de réserves vitellines plus importantes. Cette hypothèse a été vérifiée par DELPECH (I967, résultats non publiés) : des poussins, de poids identiques à la naissance, ont un sac vitellin beaucoup plus important et, par conséquent, une quantité de tissus plus faible, s'ils possèdent le gène $d w$. Le gène de nanisme affecte donc la croissance des sujets dès le développement embryonnaire, contrairement aux affirmations de Hurr (I959).

L'indice de consommation étant un assez mauvais critère de l'efficacité alimentaire, nous avons utilisé la méthode d'ARNOULD (I96I) pour distinguer le besoin énergétique d'entretien de celui de croissance. Cette méthode, rappelons-le, consiste à porter le taux d'ingestion en fonction du taux de croissance et à assimiler l'ordonnée à l'origine au besoin d'entretien tandis que la pente de la droite fournit une estima-
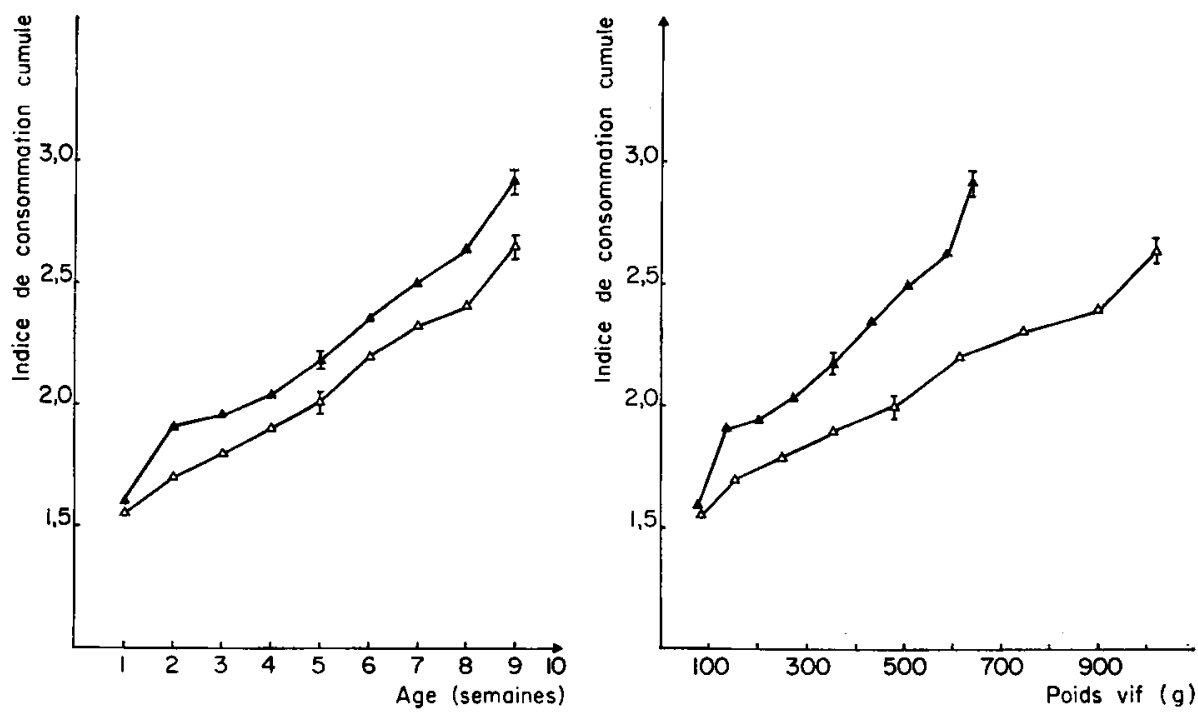

FIG. 4. - Indice de consommation de poulettes a naines "et "normales " établi sur 8 paires de vraies soeurs el de 8 demi-sours

Même convention que dans la figure I pour les symboles correspondant aux deux génotypes

tion du besoin de croissance. Nous avons montré que cette méthode, par ailleurs discutable, donne des résultats intéressants chez le Poussin sur une période assez brève de sa croissance (GUILLAUMME, I966). Dans le cas de cette expérience, cette méthode permet d'éliminer l'influence du taux de croissance sur l'indice de consommation global.

Les équations des droites de régression calculées sur les données moyennes de chaque semaine sont rapportées dans lle tableau 5 pour les deux régimes utilisés. L'utilisation de l'aliment pour la croissance seule (après déduction de l'entretien) est moins bonne chez les individus $d w$ qui demandent I6 à $26 \mathrm{p}$. Ioo de plus d'aliment par unité de croît durant le démarrage et la finition respectivement. Le besoin d'entretien, par contre, est plus faible chez les naines bien que la différence considérable au démarrage devienne infime et non significative durant la $2^{\mathrm{e}}$ période d'élevage. 
TABLEAU 5

Relation entre taux d'ingestion $\left(\frac{\mathrm{I}}{\mathrm{P}}\right)$ et taux de croissance $\left(\frac{\Delta \mathrm{P}}{\mathrm{P}}\right)$ chez les poulettes naines et normales

(23 paires de sœurs)

\begin{tabular}{|c|c|c|}
\hline & $\mathrm{D}_{w}$ & $d w$ \\
\hline $0-5$ semaines & $\frac{\mathrm{I}}{\mathrm{P}}=4,51+1,24 \frac{\Delta \mathrm{P}}{\mathrm{P}}$ & $\frac{\mathrm{I}}{\mathrm{P}}=\mathbf{3 , 5 0}+1,56 \frac{\Delta \mathrm{P}}{\mathrm{P}}$ \\
\hline 6-9 semaines & $\frac{\mathrm{I}}{\mathrm{P}}=3,00+1,73 \frac{\Delta \mathrm{P}}{\mathrm{P}}$ & $\frac{\mathrm{I}}{\mathrm{P}}=2,90+2,00 \frac{\Delta \mathrm{P}}{\mathrm{P}}$ \\
\hline
\end{tabular}

$\frac{\mathrm{I}}{\mathrm{P}}$ et $\frac{\Delta \mathrm{P}}{\mathrm{P}}$ sont obtenus en divisant l'ingéré et le crô̂t journalier moyen au cours d'une semaine par le poids vif moyen de cette semaine.

\section{I)ISCUSSION}

Habituellement les animaux à croissance rapide ont une meilleure efficacité alimentaire car la part de leur besoin d'entretien dans leur besoin total est plus faible.

En fait, cette loi n'est pas générale comme l'ont montré SIEGEL et WISMAN ( I966). Ces auteurs ont travaillé sur deux lignées d'animaux issus d'une même souche mais sélectionnés soit pour un poids vif élevé, soit pour un poids vif faible à 8 semaines. Ils ont comparé le développement pondéral, la consommation, 1'utilisation globale de la ration, l'utilisation de l'azote et de 1'énergie ainsi que 1'énergie métabolisable dans les 2 lignées. Tous ces critères d'efficacité nutritionnelle sont identiques dans les 2 lignées si les animaux sont nourris ad libitum. Une sélection modifiant la croissance n'a donc pas les mêmes répercussions sur l'utilisation alimentaire selon qu'elle porte sur un seul caractère monogénique ou sur un critère quantitatif polygénique.

Le gène $d w$ a des effets bien marqués qui se résument ainsi : réduisant la croissance sans affecter sensiblement ni la consommation, ni la teneur azotée de la carcasse, il entraîne automatiquement une détérioration de l'indice de consommation. Il abaisse la dépense énergétique d'entretien, ce qui se traduit par une certaine économie; le rapport gain de poids/énergie (ou aliment) ingérée est détérioré malgré cela par suite d'une élévation du " besoin de croissance ». L'équation d'ARNoulo (I96I) permet de chiffrer les besoins mais n'apporte pas d'explication. Grâce aux mesures de consommation d'oxygène et à l'analyse corporelle, on peut affirmer que l'accroissement du besoin de croissance est dû essentiellement à l'enrichissement du croît en matières grasses. Une légère diminution du rendement métabolique des synthèses est possible, mais si elle existe, elle est plus que compensée par l'abaissement de la dépense basale comme l'indique la valeur du métabolisme global. De tout cela, il résulte un meilleur " rende- 
ment " énergétique global qui contraste avec la moins bonne " utilisation " des protéines et le moins bon indice de consommation. Le poussin $d w$ apparait donc comme un animal à croissance ralentie, à métabolisme basal abaissé et à consommation comparable à celle du poussin normal. De ce fait, il ingère par rapport à son crô̂t plus de protéines et d'énergie que le normal. L'excédent de protéines est catabolisé, celui (plus grand encore) d'énergie est déposé dans les tissus.

Si l'on admet que les besoins azotés sont proportionnels à l'intensité de la synthèse protéique il apparaît alors que le poussin $d w$ consomme plus qu'il n'a besoin. Gaspillage des protéines de la ration et engraissement excessif sont des conséquences de la boulimie. D'autres explications sont possibles : ou bien l'adiposité des tissus résulterait d'une capacité de synthèse lipidique activée par le gène dw ou tout au moins réduite plus faiblement que ne l'est la synthèse protéique ; une surconsommation relative serait alors nécessaire à l'approvisionnement en énergie alimentaire et un gaspillage de protéines en résulterait. Ou bien, le gène de nanisme perturberait les mécanismes de synthèse protéique du Poussin qui, pour fabriquer moins, serait obligé de consommer autant qu'un normal. Ces dernières hypothèses sont bien en accord avec la loi voulant qu'un mutant soit d'abord un " anormal ". Il reste cependant à démontrer laquelle est exacte.

Un point troublant demeure : nos oiseaux $d w$ ont une plus mauvaise conversion alimentaire globale que les normaux, alors que BERNIER et ARSCOTT (I966) ont observé l'inverse sur des poulettes Leghorn. Cette divergence provient-elle d'une différence de composition dans les aliments utilisés ou d'une interaction avec le reste du génôme?

Le gène sur lequel porte la ségrégation a été trouvé par MéRAT en I959 et semble très voisin de celui décrit par HuTT (I953-I959). Il n'est cependant pas exclu qu'i1 s'agisse d'un allèle voisin (MÉRAT, I969) voire d'un autre gène (ARscoTT, I968).

Quoi qu'il en soit, plusieurs des faits mis en évidence dans cet essai : diminution de la dépense énergétique d'entretien, calme, grande adiposité des tissus sont en accord avec l'hypothèse selon laquelle nos poussins $d w$ sont hypothyroïdiens (MÉRAT et GUILLAUME, I969).

Bien que fournissant peu de renseignements sur les besoins du poussin $d w$ ces données laissent supposer qu'il réagit différemment à un changement du rapport cal/prot, ce que nous allons vérifier prochainement.

Reçu pour publication en mars 1960.

\author{
SUMMARY
}

EFFECT OF INTRODUCING THE DWARF GENE “ DW " ON FEED UTILISATION IN FEMALE CHICKENS

We compared normal and dwarf (allele $d w$ ) sister pullets from an experimental pedigree crossing. Weight gain, intake of feed and various criteria of utilisation of the feed, protein and energy were recorded. One feed, of the usual type, modified at 5 weeks, was given during the experiment which lasted 9 weeks.

Compared with normal pullets of the same weight the dwarf pullets ate as much feed and grew less quickly (fig. I). Intake of feed per unit gained was greater (fig. 4) and efficiency of utilization of protein was less (table 3). Metabolisable energy of the diet was not affected (table 4 ). 
Maintenance requirement (table 5), resting metabolism (fig. 3) and probably basal metabolic rate were reduced by the $d w$ gene. Requirement of feed for gain increased even more than total intake per unit gained, but this was essentially due to an increase of lipid content in the tissue formed. Utilisation of energy, measured as the relation of energy retained to intake of metabolisable energy, was in favour of the dwarf pullets. The modifications, in the opposite directions, of utilisation of energy and of protein are discussed in relation to the relatively more voracious nature of the $d w$ individuals. The results concerning the global efficiency of feed utilisation are contrary to those of BERNIER and ARscotT (I966).

It was noted that the dwarfs had a much more regular oxygen consumption, indicating a calmer disposition, which confirmed a subjective impression. Several of the facts observed equally suggest hypothyroidism in the dw subjects.

\section{RÉFÉRENCES BIBLIOGRAPHIQUES}

ARNould R., 1961. L'utilisation des protéines pour la croissance. Thèse Université de Louvain (Belgique). ArscotT G. H., I968. Communication personnelle.

ARscotT G. H., BERnier P. E. 1968. Effect of dietary protein on performance of dwarf White Leghorn layers. Poull. Sci., 47, $195^{2}$.

Bernier P.E., ARscort G. H., ig6o. Relative efficiency of sex-linked dwarf layers and their normal sisters. Poult. Sci., 39, 1 234-1 235.

Bernier P. E., ARscotT G. H., 1966. Growth and feed requirements of dwarf White Leghorn pullets as compared to their normal size sisters. Poult. Sci., 45, 1070.

Delpech P., Guezel. M., Leclerce B., Kahane E., 1966. Méthode d'extraction des lipides en continu et à chaud par le mélange azéotrope : Benzène-Éthanol-Eau. Rev. Fr. Corps gras, 13, 61 5-620.

Guillaume J., rg66. Le rôle des protides dans l'utilisation des aliments du Poussin. III. Effets du taux protidique sur l'indice de consommation. Ann. Biol. anim. Bioch. Biophys., 6, 4I I-421.

GUILlaUME J., I969. Une technique entièrement gravimétrique de mesure des échanges respiratoires en circuit fermé pour petits animaux. A paraître.

Hill F. W., ANDERson D. L., 1958. Comparison of metabolizable energy and productive energy determinations with growing birds. J. Nutr., 69, $587-603$.

Hutt F. B., 1953. Sex-linked dwarfism in the fowl. Genelics, 38, 670.

HutT F. B., 1959. Sex-linked dwarfism in the fowl. J. Heredity, 40, 209-221.

MÉrat Ph., I 969 . Étude d'un gène de nanisme lié au sexe chez la Poule. I. Description sommaire et performance. Ann. Génét. et Sélect. anim., 1, 19-26.

Mérat Ph., Guillaume J., 1969. Nanisme lié au sexe et fonctionnement thyroidien chez la Poule. Ann. Génet. et Select. Anim. Sous presse.

Prod'homme J., Mérat Ph., I 969 . Consommation alimentaire et production de poules de génotypes $\mathbf{D} w$ et $d w$ suivant la teneur en calcium de la ration. Ann. Génét. Sélect. anim. (sous presse).

Siegel P. B., Wisman E. L., 1966. Selection of body weight at 8 weeks of age. 6. Changes in appetite and feed utilization. Poult. Sci., 45, 1391-1 397 . 